\title{
La complejidad del proceso de conocimiento
}

Luis Armando González ${ }^{1}$

Recibido en Septiembre 2011, aprobado en Octubre 2011

"El conocimiento científico aspira a ser totalmente impersonal y trata de formular lo descubierto por el intelecto colectivo de la humanidad"

Bertrand Russel

"La ciencia busca el movimiento perpetuo [...] Todo se mueve en ella, todo cambia, todo se despoja de su piel vieja".

Víctor Hugo

\section{Resumen}

En este artículo se hace un análisis de la complejidad y su vinculación con la realidad, con el conocimiento y con su asimilación y con la puesta en práctica de dicho conocimiento.

\section{Palabras clave:}

Conocimiento, complejidad, teoría, praxis, realidad, transformar la realidad, emergencia.

\author{
Abstract \\ In this article the author analyzes the complexity and its relation \\ with reality, with knowledge and its assimilation, and with the \\ actual practice of that knowledge
}

Keywords:

Knowledge, complexity theory, praxis, reality, transformation of reality, emergency. 


\section{Introducción}

El conocimiento es un proceso complejo no sólo en su punto de partida -la subjetividad humana y los procesos neuropsicológicos que en ella se hacen presentes en tanto se conoce- sino en su punto de llegada -la realidad social y natural con sus dinamismos y estructuras intrínsecos-. Todavía no ha sido dicha la última palabra acerca los factores que internamente -en la subjetividad humana- dan vida al conocimiento. Sabemos que son neurofisiológicos y simbólicos; que en ellos el lenguaje es crucial, pero también la acomodación psicobiológica, por parte del individuo, a su entorno socio-natural. El tema mente-cuerpo acecha a cada paso, sin que se tengan respuestas definitivas ante el mismo. Y es que, como dijo hace casi 20 años J. L. González Quirós,

“necesitamos algo que -a punto entrar en lo que algunos han comenzado a llamar el siglo del cerebro- no parece echarse suficientemente en falta: una teoría coherente de la mente. El hecho de que carezcamos de ella sirve de obstáculo para ocultar deficiencias muy graves en nuestra imagen de lo real que, como es frecuente, se tratan de paliar mediante procedimientos expeditivos que no deberían considerarse de recibo: bien con un exceso de detalles que nadie se molesta en precisar, o bien suponiendo, con suficiencia postmetafísica, que se trata de cuestiones que nuestra superior perspicacia ha acertado a abandonar. Sin embargo, no es preciso mucho tiempo para comprender que existen lagunas y contradicciones en nuestras actuales teorías de lo mental. Tales carencias no dejan de existir porque no sean sometidas a consideración: son paradojas y ambigüedades que ningún prejuicio brillante puede ocultar del todo"2.

Queda mucho por explicar sobre los fundamentos biológicos del conocimiento, especialmente esa delicada imbricación entre sistema nervioso superior y las ideaciones, la memoria, el análisis y la reflexión ${ }^{3}$. No queda duda de que se trata de una imbricación compleja que, para ser explicada, requiere del mejor esfuerzo cognoscitivo del ser humano, esfuerzo en sí mismo complejo en el que justamente entran en juego los dinamismos que se están pretendiendo conocer. Sólo al ser humano le ha sido dada la capacidad -por razones evolutivas y socioculturales- de poner en juego sus propias capacidades cognoscitivas para conocer el proceso de conocimiento del cual el mismo es autor ${ }^{4}$.

\section{Conocimiento y realidad}

Visto desde la interioridad subjetiva y psicobiológica del ser humano, el conocimiento no es fácil de comprender. Tampoco lo es desde la realidad que

2 J. L. González Quirós, Mente y cerebro. Madrid, Iberediciones, 1994.

3 Cfr. K. R. Popper, J. C. Ecles, El yo y su cerebro. Barcelona, Roche, 1980.

$4 \mathrm{Cfr}$., H. Maturana y F. Varela, El árbol del conocimiento. Las bases biológicas del conocimiento humano. Madrid, Debate, 1996. 
se quiere conocer. El mismo conocimiento que se tiene de la realidad -lo cual se ha probado de muchas maneras- apunta hacia algunas conclusiones no definitivas ni últimas, pero sí firmes, en torno a la misma. Una de ellas es que la realidad es compleja, lo cual quiere decir que se articula en distintos niveles de estructuración ${ }^{5}$, con ámbitos y dimensiones cuya articulación está más allá de lo evidente, con momentos de azar y caos no esporádicos o accidentales, sino intrínsecos ${ }^{6}$.

Y es que "ya se trate de la química del cosmos, la química de la Tierra, la química de la vida, la historia del universo, la historia de los planetas o la historia de la vida, en todos los anales -referentes a la materia o a la vidagobierna la contingencia. Nada indica una programación de ningún tipo, un libreto inevitable, un recorrido previsto. La Tierra es un accidente de la historia del cosmos, lo mismo que el surgimiento del hombre. El sentido de la historia no es más que una ilusión a posteriori, como lo es la supuesta teoría antrópica. Nada en el estudio científico de los hechos permite entrever un determinismo histórico de ningún tipo, ni en la evolución de la naturaleza ni en la de la sociedad"'.

La realidad, pues, es compleja. Uno de los autores que más han explorado esa complejidad de la realidad es Ilya Prigogine, quien nos dice que

"hay que pensar el universo como una evolución irreversible; la reversibilidad y la simplicidad clásicas resultan entonces casos particulares [...]. Las grandes líneas de la historia del universo están hechas de una dialéctica [...] entre la gravitación y la termodinámica, o, si se quiere, entre Einstein y Boltzman [...]. El futuro del universo no está determinado de ninguna manera [...]. A mi entender, el mensaje que lanza el segundo principio de la termodinámica es que nunca podemos predecir el futuro de un sistema complejo. El futuro está abierto y esta apertura se aplica tanto a los sistemas físicos pequeños como al sistema global, al universo en que nos encontramos [...]. Con la aparición de la vida, nace un tiempo interno que prosigue durante los miles de millones de años de la vida y se transmite de una generación a otra, de una especie a otra especie, no sólo se transmite, sino que se hace cada vez más complejo"8.

5 X. Zubiri, Estructura dinámica de la realidad. Madrid, Alianza, 1989.

6 Cfr. M. Bunge, "Azar y casualidad, caos y accidente”. En Cápsulas. Barcelona, Gedisa, 2003, pp. 77-80.

7 C. Allégre, La derrota de Platón o la ciencia en el siglo XX. México, FCE, 2003, p. 223.

8 I. Prigogine, El nacimiento del tiempo, Barcelona, Tusquets, 1998 , pp. 25 y ss. En su texto clásico, El fin de las certidumbres (Madrid, Taurus, 1997), Prigogine sostiene que "nuestro mundo es fluctuante, caótico, más semejante al que los atomistas griegos habían imaginado" (p. 146). Ver también, I. Prigogine, El nacimiento del tiempo. Barcelona, Tusquets, 1998; P. Smith, El caos. Una explicación de la teoría. Madrid, Cambridge University Press, 2001. 
El ser humano y su conocimiento son expresión de esa complejidad de lo real. La realidad reclama que el proceso de conocimiento sea complejo, pues de lo contrario se daría un desfase que dejaría al ser humano al margen de ella, poniendo en tela de juicio su propia supervivencia como especie. Evolutivamente, el ser humano ha respondido a esa complejidad de lo real con una complejización creciente del proceso de conocimiento, lo cual está sostenido por la complejidad de su sistema nervioso superior ${ }^{9}$. El ser humano ha avanzado de lo aparente - que es lo más simple- a lo esencial. Ha avanzado de lo concreto a lo abstracto. Partiendo de lo que se sabe ha avanzado hacia lo que no se sabe, pero también desde lo que no sabe ha interrogado a lo que se sabe. Con una lucidez impresionante, Parménides (515-450 a C.) planteó el enorme desafío que supone para el conocimiento vencer lo aparente que se nos da a través de los sentidos,

"lo que está en cualquier momento en los engañosos órganos de los sentidos, eso les parece a los hombres conocimiento genuino, pues tienen por lo mismo la mente intelectual del hombre y la cambiante naturaleza de sus órganos de los sentidos.

Llaman 'pensamiento' a lo que prevalece de este embrollo en todos y cada uno de los hombres".

"Nunca se concederá que las cosas que no son existan.

Aparta tu pensamiento de esta vía de indagación; no permitas que la experiencia y la rutina se te impongan. $Y$ no dejes errar tus ciegos ojos o tus sordos oídos, ni siquiera tu lengua, por esa vía”.

Con el conocimiento científico el ser humano ha respondido a la complejidad de la realidad. ¿De qué manera? Trascendiendo los hechos, descartando hechos, produciendo nuevos hechos y explicándolos. Trascendiendo el sentido común que "parte de los hechos y se atiene a ellos: a menudo se limita al hecho aislado, sin ir muy lejos en el trabajo de correlacionarlo con otros o de explicarlo [...]. La investigación científica no se limita a los hechos observados: los científicos exprimen la realidad a fin de ir más allá de las apariencias"10.

Ciertamente, no se ha tratado de algo fácil, pues durante mucho tiempo se creyó que la tarea de la ciencia era simplificar lo complejo de la realidad. Según J.- M. Lévi-Leblond, la física surgió dando la espalda a la complejidad, "pero la realidad es compleja, aun cuando la física se esfuerce en pasarlo por alto" ${ }^{11}$. Así que la física ha tenido que complejizarse, para ponerse a tono con lo real: "esa complejidad de la estructura teórica de la física responde a lo real, en que

9 Cfr. A. R. Luria, El cerebro humano y los procesos psíquicos. Barcelona, Fontanella, 1979. 10 M. Bunge, La ciencia, su método y su filosofía. Santafé de Bogotá, Panamericana, 1996, p. 22. $11 \mathrm{~J}-\mathrm{M}$. Levy-Leblond, La piedra de toque. La ciencia a prueba. México, FCE, 2004, p. 252. 
coexisten e interactúan lo global y lo local, el movimiento y la permanencia, lo elemental y lo compuesto, lo finito y lo infinito, etc."12. También se creyó, desde un inductivismo ingenuo, que la tarea de la ciencia consistía en recolectar datos, organizarlos, clasificarlos o tabularlos y desde ahí realizar generalizaciones que expresaran "leyes generales". Las falencias del inductivismo absoluto son evidentes, de tal suerte que quienes pretenden justificarlo como la vía correcta para aproximarse a la realidad son presa fácil de la crítica. Ir a la realidad sin más armas que los sentidos no sólo es una empresa imposible, sino que cuando se intenta revela una pobreza epistemológica de enormes proporciones. Para conocer científicamente la realidad, las apariencias, que son precisamente lo que se obtiene a través de los sentidos, tienen que ser vencidas. Y no con un deductivismo absoluto, sino con una sabia articulación de teoría y experiencia, como bien lo entendió Kant. Mario Bunge lo explica de este modo,

"la ciencia no es un una mera prolongación ni un simple afinamiento del conocimiento ordinario, en el sentido de que el microscopio, por ejemplo, amplía el ámbito de la visión. La ciencia es un conocimiento de naturaleza especial: trata primariamente, aunque no exclusivamente, de acontecimientos inobservables e insospechados por el lego no educado; tales son, por ejemplo, la evolución de las estrellas y la duplicación de los cromosomas; la ciencia inventa y arriesga conjeturas que van más allá del conocimiento común, tales como las leyes de la mecánica cuántica o de los reflejos condicionados; y somete esos supuestos a contrastación con la experiencia con ayuda de técnicas especiales, como la espectroscopia o el control del jugo gástrico, técnicas que, a su vez, requieren teorías especiales"13.

En definitiva, el proceso de conocimiento es complejo por dondequiera que se le mire. Es complejo en su dinámica estrictamente cognoscitiva -es decir, en lo que atañe a los procesos conceptuales que involucra-como en lo que se refiere a los procesos neuropsicológicos involucrados. Ni sobre lo uno ni sobre lo otro hay estudios -conocimientos-concluyentes. Ni mucho menos sobre la relación entre ambos órdenes de realidad. Así, por más que las neurociencias estén de moda -estén aportando conocimientos significativos sobre las redes neuronaleses discutible identificar procesos mentales con procesos neuronales ${ }^{14}$, lo cual puede llevar a la tesis de que lo mental (ideas, creencias, conceptos, palabras, etc.) son una segregación electroquímica del cerebro. Claude Allégre, a

\section{Ibid., p. 254.}

13 M. Bunge, La investigación científica. Barcelona, Ariel, 1979, p. 20.

$14 \mathrm{~A}$ ratos, pareciera ser que Mario Bunge se mueve en esa dirección, cuando escribe "si los procesos mentales no son procesos neurológicos, ¿cómo se explica que el cerebro sea el más complicado y vulnerable de todos los órganos? Si no cumpliera las funciones más exquisitas que cabe imaginar, tales como inventar dioses y derrocarlos, ¿por qué no bastaría una cabeza hueca, útil solamente para llevar sombrero y cabecear pelotas de fútbol”. M. Bunge, "El papa y la evolución”. En Cápsulas..., pp. 115-116. 
quien ya citamos, defiende, desde una visión exageradamente optimista de las capacidades explicativas de las neurociencias, la tesis de que los objetos mentales son "grafos de representación" elaborados neuronalmente.

En esta línea, el cerebro humano (en su carácter de estructura neuronal) sería el agente central y casi exclusivo de la vida mental: inventa realidades, crea ficciones, imagina $\cos ^{15}{ }^{15}$. En el límite, siempre en el terreno de la vida mental, lo que se afirma es la "realidad física de los objetos mentales"16. Y, de cara a la sociedad, lo que se sostiene es que las sociedades son "superredes neuronales formadas por la asociación de millares o millones de cerebros que intercambian información"17.

Suponiendo que lo del intercambio de información sea cierto, ¿de dónde emerge, dónde se genera esa información? Y sus contenidos simbólicos -por ejemplo la información (lo que se sabe) sobre la estructura y funciones del cerebro- ¿es una realidad física? ¿Es una segregación electroquímica del cerebro? Aquí hay mucho que debatir, sin que ello suponga negar la base material (neurofisiológica) de los procesos mentales. Lo mismo que cabe discutir la afirmación -siempre de C. Allégre- de que las ideas, los conceptos no son inmateriales. Choca contra la razón no aceptarlo, pues es difícil entender y probar la materialidad de la idea de mesa, de la idea de silla o de la idea de cerebro -que no son equivalentes a la mesa, la silla o el cerebro materiales-. Aceptar la inmaterialidad de las ideas (o las creencias y los conceptos) no significa que las ideas "floten en el aire" sin ningún soporte físico, sino que no se reducen a su soporte neurológico.

En este punto, es esclarecedor el concepto de "emergencia", usado por Mario Bunge para referirse a procesos novedosos cuantitativos o cualitativos resultantes de "combinaciones de unidades de inferior nivel [que] poseen propiedades de las cuales sus partes o precursores carecen"18. Asimismo, "no hay emergencia en sí misma o separada de las cosas que emergen: sea lo que fuere que emerge, su surgimiento tiene lugar en un objeto [...]. Y no hay emergencia ex nihilo: todo emerge a partir de algo, como las interacciones o bien entre los componentes de un sistema o bien entre algunos de ellos y elementos del entorno. De este modo, le refracción emerge en un medio a partir de la interacción de este con la luz; y el lenguaje emerge en las cabezas de los niños que interactúan con otros seres humanos"19. Este punto der vista emergentista se distancia de las visiones que "intentan reducir los fenómenos mentales a fenómenos neuronales", es decir, que sostienen que "los fenómenos

15 Cfr. F. Martínez Mendoza, Neurociencias y formación inicial. México, Trillas, 2010.

16 C. Allégre, La derrota de Platón..., p. 215.

17 lbíd., p. 218.

$18 \mathrm{M}$. Bunge, Emergencia y convergencia. Novedad cualitativa y unidad del conocimiento. Barcelona, Gedisa, 2003, p. 29.

19 Ibíd., p. 35. 
mentales son fenómenos cerebrales"20. "Muchos neurocientíficos, incluido quien esto escribe -dice F. J. Álvarez Leefmans-, consideramos que las funciones mentales, la conciencia entre ellas, son propiedades emergentes del sistema nervioso. El término emergente aquí no tiene ninguna connotación mística, religiosa, milagrosa o esotérica, simplemente significa que la conciencia es una propiedad del sistema como un todo y no de las partes que lo constituyen" ${ }^{21}$.

Hemos esbozado las grandes líneas epistemológicas en las que la complejidad del proceso de conocimiento es clara. Si se aterriza a aspectos más puntuales, dicha complejidad también se hace presente.

\section{La asimilación cognoscitiva y su temporalidad}

Reflexionemos sobre la asimilación de nuevos conocimientos. Aunque ha habido situaciones históricas en las cuales los individuos, con su particular bagaje de conocimientos, se han visto poco expuestos al desafío de conocimientos nuevos -o los conocimientos adquiridos los han blindado para asimilar otros nuevos-, desde la época moderna lo normal es que, sin importar el bagaje cognoscitivo que se tenga, el mismo puede ser desafiado por nuevos conocimientos. El nacimiento y auge de la ciencia en la modernidad ha sido clave en la implantación de esta forma de proceder en el proceso de conocimiento; el clima cultural de la modernidad, al incorporar el ethos científico, incorpora la vocación por la revisión y crítica de los conocimientos adquiridos. Y es que la ciencia se caracteriza por exigir, desde sí misma, el reemplazo de viejos conocimientos por nuevos. "Tan pronto -escribe Mario Bunge- como ha sido establecida una teoría científica, corre el peligro de ser refutada o, al menos, de que se circunscriba su dominio. Un sistema cerrado de conocimiento fáctico, que excluya toda ulterior investigación, puede llamarse sabiduría pero es en rigor un detritus de la ciencia. El sabio moderno, a diferencia del antiguo, no es tanto un acumulador de conocimientos como un generador de problemas. Por consiguiente, prefiere los últimos números de las revistas especializadas a los manuales, aun cuando estos últimos sean depósitos de verdad más vastos y fidedignos que aquéllas. El investigador moderno ama la verdad pero no se interesa por las teorías irrefutables"22.

Este proceder científico ha salido extramuros de las academias y se ha convertido, hoy por hoy, en algo actuante en la cultura mundial. Así, no sólo es normal que el acervo de conocimiento con el que se cuenta sea puesto a prueba permanentemente por distintas instancias del entorno en el que se mueve el individuo, sino que la sociedad moderna ha generado, en el plano subjetivo, una actitud de buscar nuevos conocimientos, de no conformarse con

20 F. J. Álvarez Leefmans, "La emergencia de la conciencia". En R. de la Fuente y J. Álvarez Leefmans, Biología de la mente. México, FCE, p. 69.

21 lbíd., p. 68.

22 M. Bunge, La ciencia, su método y su filosofía. Santafé de Bogotá, Panamericana, 1996, p. 42. 
lo sabido, de confirmar o de someter a prueba lo que se sabe. Los niveles de sistematización y profundidad varían en cada cual, pero la actitud está ahí, anclada en un impulso que nace de lo profundo de las estructuras de la psique humana. En otras palabras, la sociedad moderna está marcada por las ansias de buscar certeza, sin nunca encontrarlas. El motor de esas ansias -no ajeno a la ciencia- es la convicción afianzada en el psiquismo del individuo moderno de que no hay verdades científicas irrefutables, sino que toda verdad científica es una conjetura y una aproximación a la realidad. Es decir, que todo saber científico es provisional y que el camino hacia el conocimiento de la realidad está salpicado de errores y equivocaciones, más que de aciertos. En definitiva, que refutación de las teorías vigentes es la mejor herramienta que poseemos para avanzar en la aprehensión intelectual de la realidad ${ }^{23}$.

Ahora bien, confrontarse con nuevos conocimientos es una cosa; otra muy distinta es asimilarlos, vale decir, integrarlos -si son coherentes- en el propio andamiaje de conocimientos previos o superar, con su ayuda, aquello que aporta menos a la aprehensión intelectual de la realidad. Ambos aspectos son difíciles para quien se ve afectado por ellos. No es fácil integrar nuevos conocimientos en el saber previo que se posee; mucho menos lo es romper parcial o totalmente con este último. Cuando esto sucede se asiste a situaciones de crisis intelectual (moral y ética, en muchos casos) que pueden ser traumáticas para quienes las viven. De hecho, lo que Thomas Kuhn llamó "revoluciones científicas" tiene un componente inevitable de crisis intelectual individual, que se explica justamente por la ruptura con los paradigmas establecidos propia de una revolución científica ${ }^{24}$. En el ámbito de la teología, la noción de "conversión" apunta nítidamente a este proceso de crisis intelectual y moral, así como al nuevo posicionamiento personal que se genera después de ella.

Desde el punto de vista del estudioso de estos procesos, su complejidad es lo que se impone irremediablemente. Durante mucho tiempo se creyó que el asunto era mecánico: un agente externo (un profesor, una lectura, etc.) ponía a disposición de un individuo (entendido como un receptor pasivo) un nuevo conocimiento; este último -una vez que lo veía, leía o escuchabase lo apropiaba (repetía la lección o el texto para "probar" cuánto sabía) y así, sin tanta complicación, se presumía que sabía un poco más, que había aumentado su conocimiento. Si él quería "transmitir" ese conocimiento a otros sólo tenía que repetir el mismo procedimiento. Es una visión escolar -bancaria, la llamó Paulo Freire- del proceso de transmisión de conocimiento. Se trata de una concepción escolástica, fraguada en la edad media y que llegó a tierras americanas con la conquista y la colonización española y portuguesa. El ensayista y poeta mexicano, Octavio Paz, lo destacó bien cuando señaló, en su libro Sor Juana Inés de la Cruz o las trampas de la fe, que lo propio de 
la educación colonial fue hablar, desde la cátedra, hasta la sociedad, a unos alumnos que escuchaban, apuntaban y luego repetían la lección.

Comprender el proceso de asimilación de nuevos conocimientos no es sencillo. Los conocimientos previos pueden favorecer esa asimilación, pero pueden ser una barrera difícil de vencer. Es decir, el individuo que confronta su bagaje cognoscitivo con nuevos conocimientos puede tener fuertes resistencias mentales - "blindajes psicológicos"- para integrarlos, pero también valerse de ellos para superar sus conocimientos más débiles o menos fundamentados. Se trata de un proceso subjetivo e intersubjetivo, del cual la repetición de un discurso o unas fórmulas nos dice bien poco. Por lo mismo, cualquier medición que se haga de la adquisición de nuevos conocimientos siempre será insatisfactoria, especialmente si no logra captar que esa asimilación (o no asimilación) es inseparable de la lucha que cada quien libra para integrarlos en el marco de los conocimientos adquiridos.

En la asimilación de nuevos conocimientos se trata, asimismo, de un proceso que puede tener una duración temporal variable, pero que en todo caso no es algo inmediato. No es que ahora un individuo se las ve con un nuevo conocimiento y mañana ese nuevo conocimiento ya es parte de su bagaje cognoscitivo. Debe pasarlo por el tamiz de lo que ya sabe; examinar lo que sabe a la luz de lo nuevo; renunciar a "conocimientos viejos", para poner en su lugar los nuevos conocimientos adquiridos; y finalmente alcanzar una articulación coherente (sistémica) de los distintos conocimientos que posee: esto último significa tener una cosmovisión, es decir, una visión coherente del mundo.

Frecuentemente, en el tema del conocimiento, la variable temporal se escamotea, pero es en el tiempo que el proceso de conocimiento se decanta, madura y se reproduce. Olvidar el tiempo trae consigo el vicio del inmediatismo, que consiste en no valorar el conocimiento bien cimentado en las estructuras psicológicas de los individuos, en valorar lo de corto plazo y lo efímero. En menospreciar aquello que sólo dará frutos en el mediano y largo plazo porque antes requiere de una maduración inevitable. En frustrarse cuando, al siguiente día de un esfuerzo intelectual realizado, básicamente las cosas siguen igual ya que ese esfuerzo fue uno -pequeño, pero parte de un andamiaje mayor- de una serie sostenida de esfuerzos intelectuales.

\section{El ethos científico}

Nada de lo anterior es mecánico o inmediato. Es sumamente inseguro en personas no educadas adecuadamente para ello. Es decir, la profundización, avance y superación del propio conocimiento es asunto de educación. $Y$ de un tipo de educación en particular: la científica, entendida no de manera cientificista y positivista, sino de modo crítico. Porque algo connatural al quehacer científico 
es precisamente el cuestionamiento, revisión, puesta a prueba y superación del conocimiento que, con ese quehacer (teórico y experimental), se va logrando progresivamente. Es también connatural a la ciencia dar sostén a "concepciones del mundo que concuerdan con los hechos" y crear el hábito de "adoptar una actitud de libre y valiente examen, en que acostumbra a la gente a poner a prueba sus afirmaciones y a argumentar correctamente"25. Los hábitos dogmáticos y fundamentalistas son reacios a lo anterior; como lo son los hábitos anclados en el cortoplacismo, el inmediatismo y las apariencias. El conformismo y el conservadurismo tampoco son coherentes con el ethos científico.

Que la ciencia entienda su conocimiento de la realidad como provisional, conjetural o hipotético es indicativo del espíritu crítico que la anima. Quien se educa según las exigencias epistemológicas de la ciencia se prepara para someter, de manera sistemática, sus conocimientos al desafío de nuevos conocimientos; más aún, se prepara para buscar nuevos y mejores conocimientos con los que superar los que posee. $Y$ esta búsqueda no sólo es teórica; es también experimental. Obviamente, los científicos de profesión son los más diestros en este proceder, pero aspectos importantes del ethos científico pueden ser -deberían ser- asumidos por quienes no lo son.

De hecho, una de las conquistas irrenunciables de la cultura moderna -en lo cual los sistemas educativos jugaron un papel decisivo- es haber permitido que este ethos se difundiera en diferentes ámbitos sociales. Sin embargo, pese a los más de tres siglos de progresivo desarrollo científico -si arrancamos de las contribuciones de Isaac Newton (1646-1727)-, el "espíritu científico" (G. Bachelard) ni es compartido íntegramente por todos los sectores sociales ni lo está con igual rigor y sistematicidad en los ámbitos en los que está presente. De lo que resulta que amplios grupos sociales, pese a no ser ajenos a algún tipo de irradiación del espíritu científico, tienen serias dificultades para asimilar nuevos conocimientos y peor aún para superar su bagaje cognoscitivo con uno nuevo. No entender esas dificultades puede llevar a la creencia ingenua de que basta con que alguien esté "expuesto" a una avalancha de nuevos conocimientos para que estos se conviertan, automáticamente, en parte de su acervo intelectual. Más aún, sería ingenuo pensar que el ethos científico impone su racionalidad en el mundo moderno.

Con un enorme sentido crítico hacia la ciencia, Jean-Marc Levy-Leblond sostiene que "la difusión de la ciencia nunca ha dispuesto de tantos medios (medios masivos de comunicación, libros, museos, etc.), pero la racionalidad científica sigue estando amenazada, aislada e impotente ante ideologías que la rechazan o, lo que es peor, la recuperan [...]. Los conocimientos científicos, incluso los clásicos, no forman parte del saber común. Sus avances conceptuales, sus 
apuestas intelectuales, escapan cada vez más a los propios profesionales de la cultura" ${ }^{26}$. Y ahí donde se hacen presentes están integrados en un bloque mental que mezcla saberes, creencias y concepciones de la más distinta procedencia y calidad. En un bloque en el cual la charlatanería -tipo la cienciología- se mezcla con aspectos serios del saber; en un bloque en el cual los gurús de las pseudociencias (astrólogos, guías espirituales y expertos en motivación) se codean con científicos serios y respetados.

\section{Aplicación práctica del conocimiento}

Se ha tocado hasta este momento el tema de la asimilación de nuevos conocimientos. No se ha dicho nada de la aplicación práctica de ese conocimiento. Aquí la complejidad aparece de nuevo y también la temporalidad. Entre la asimilación de un nuevo conocimiento -suponiendo que esto se logre con éxitoy su aplicación hay un trecho temporal que no siempre se recorre. Recorrerlo lleva tiempo.

Asimilar nuevos conocimientos no significa que se terminen por llevar efectivamente a la práctica; o que, si intenta esa aplicación, los conocimientos adquiridos se traduzcan en su totalidad en ella. Es el viejo y debatido tema de las relaciones entre teoría y práctica, acerca del cual no se ha dicho -ni se dirá nunca-la última palabra. El denso libro del filósofo hispano mexicano Adolfo Sánchez Vázquez, Filosofía de la praxis ${ }^{27}$, explora esta compleja cuestión, de la cual siempre quedan aristas por descubrir y debatir. Pero Sánchez Vázquez lo que hace es continuar una dilatada línea de reflexión en torno a esa problemática iniciada en el siglo V antes de Cristo en la Grecia clásica.

Teoría y práctica -se dice frecuentemente- van juntas. Lo que no se dice es que el estatus ontológico de cada una es distinto: la primera pertenece al ámbito de las creaciones simbólicas; la segunda al terreno de las realizaciones operativas. Claro está que el aterrizaje práctico del conocimiento es importante, pues de lo que se trata es de la transformación de la realidad teniendo en la mira el mejoramiento de la vida humana. Pero el paso de lo simbólico a lo práctico no es automático ni inmediato: se requiere de una mediación tecnológica. 0 lo que es lo mismo, de instrumentos técnicos que hagan posible la aplicación de la teoría en la realidad. "Es redundante -dice Mario Bunge- exhortar a los científicos a que produzcan conocimientos aplicables: no pueden dejar de hacerlo [...]. La técnica precientífica era primordialmente una colección de recetas pragmáticas no entendidas, muchas de las cuales desempeñaban funciones de ritos mágicos. La técnica moderna es, en medida creciente -aunque no exclusivamenteciencia aplicada"28.

26 J-M. Levy-Leblond, La piedra de toque. La ciencia a prueba.., pp. 19-29.

27 A. S. Vázquez, Filosofía de la praxis. México, Grijalbo, 1967.

28 M. Bunge, Ibíd., p. 44. 
Ahora bien, sin las herramientas adecuadas y sin las destrezas pertinentes es imposible la aplicación práctica del conocimiento científico. Lo primero es un asunto de recursos tecnológicos, cuya ausencia o limitación (se puede tratar también de obsolescencia) puede ser un obstáculo infranqueable para el aterrizaje práctico del conocimiento.

Lo segundo es un asunto de "conocimientos técnicos" que, aunque relacionados, no son idénticos a los "conocimientos teóricos". Así, en las ciencias sociales -en otras disciplinas también se pueden encontrar ejemplos ilustrativosuna cosa es comprender teóricamente el significado y valor de distintas estrategias metodológicas para acercarse a un fenómeno social, y otra saber aplicar los instrumentos -encuestas, entrevistas en profundidad, diario de campo, observación participante, etc. - a que dan lugar esas estrategias. Se trata de saberes distintos. A menudo se confunden y eso da lugar a terribles deficiencias en el planteamiento estratégico del proceso investigativo. Técnicas de investigación y metodología no son lo mismo: esta última tiene un anclaje teórico, mientras que aquéllas tienen un anclaje instrumental.

Quizás haya quienes dominen a cabalidad ambas áreas del conocimiento. Quizás haya quienes dominen con solvencia los primeros, pero que flaqueen en los segundos. En este segundo caso, esa debilidad en el "saber técnico" -en casos extremos no se tratará de debilidad sino de ausencia- será una verdadera traba para la traducción práctica del conocimiento. Dicho de otra manera, para aplicar el conocimiento adquirido también se requiere de educación. Se entiende, de una educación específica: una que otorgue un "saber técnico"; es decir, un saber de destrezas, un saber hacer.

Por lo anterior, así como no es realista esperar que una persona asimile mecánicamente e inmediatamente un nuevo saber al que se ve enfrentada, también no es realista pensar que una persona que ha asimilado nuevos conocimientos esté lista, inmediatamente, para aplicarlos en la realidad. En el primer caso, la asimilación cognoscitiva no es mecánica sino, quizás, dialéctica. Y no es lamentable que no sea mecánica: no tiene que serlo. Tampoco lo es que no sea inmediata: por ser un proceso, tiene un ritmo temporal necesario.

En el segundo caso, la mediación tecnológica entre teoría y práctica hace imposible una conexión inmediata entre ambas. De aquí que, cuando una persona adquiere determinados conocimientos, lo que sigue no es la constatación de cuándo los aplica, sino el establecimiento de las condiciones tecnológicas que lo permitan, al igual que su adiestramiento en el manejo de esas condiciones.

\section{5. Última etapa del proceso: la enseñanza a otros}

Finalmente, una situación que no debe escapar a la reflexión es aquella en la cual una persona que ha asimilado determinados conocimientos debe enseñarlos 
a otra. De nuevo, se trata de proceso caracterizado por la complejidad y no por la simplicidad.

Partiendo del supuesto de que quien tiene la tarea de enseñar a otros ha integrado en su acervo cognoscitivo nuevos conocimientos, es incorrecto asumir que, automáticamente, está preparado para transmitirlos o ayudar a que otros los asimilen. Una cosa es apropiarse de unos determinados conocimientos -lo cual tiene su propia complejidad, como ya vimos- y otra muy distinta ponerlos a disposición de otras personas, no de cualquier manera sino en el marco de una dinámica de enseñanza.

Lo segundo no se desprende mecánicamente de lo primero, pues saber algo no significa saberlo enseñar o enseñarlo bien a otros. $Y$ es que quien ha asimilado unos determinados conocimientos y pretende enseñarlos a otros debe hacer una "traducción" de ellos en vistas a compartirlos con los destinatarios de esa traducción. Es decir, nadie transmite sus conocimientos tal como se los apropió originalmente; los traduce y adapta según sus propias capacidades y según el o los destinatarios que tiene ante sí.

A esa preparación previa -verdadero procesamiento intelectual- de lo que se sabe para ser enseñado a otros, sigue su enseñanza efectiva, momento en el cual son decisivas las destrezas pedagógicas y didácticas -que, por su parte, requieren de un aprendizaje especial-. Si estas destrezas son insuficientes el saber que se pretende compartir no sólo puede llegar distorsionado a sus destinatarios, sino que puede serles inaccesible. Obviamente, no basta con esas destrezas para enseñar bien: para enseñar bien, antes se tiene que conocer bien lo que se va a enseñar.

Ahora bien, incluso suponiendo que todos los requisitos anteriores se cumplieran a cabalidad, no se debe olvidar que los destinatarios de un proceso de enseñanza no son meros receptores pasivos de lo que le llega del exterior, sino agentes activos que, en casos extremos, podrán resistir fuertemente el embate de nuevos conocimientos. Obviando esta situación extrema, ellos tendrán su ritmo de asimilación cognoscitiva y su propio tiempo para llevar a la práctica el nuevo conocimiento asimilado. De nuevo, nada de esto es inmediato y mecánico. No tiene que serlo. Como dice Emilia Ferreiro, refiriéndose al proceso educativo con niños -pero su hipótesis vale también para los adultos-, "el niño es un interpretador, un continuo interpretador de datos. Y como la asimilación es interpretación, cuando algo se asimila es porque se le otorga significado"29.

Y es que cualquier señal que indique resultados mecánicos o inmediatos en un proceso formativo -en el que están en juego nuevos conocimientos- carecerá de seriedad. Lo normal e incluso deseable será carecer de esos resultados, en

29 E. Ferreiro, Cultura escrita y educación. Conversaciones con Emilia Ferreiro. México, FCE, 1999, p. 126. 
tanto que los que valdrán la pena serán los que revelen cambios en la visión de mundo establecida y en el posicionamiento de los actores sociales ante la realidad que les circunda. También será relevante valorar su intervención práctica en la realidad -a la luz de criterios no sólo científicos, sino éticospara transformarla y humanizarla.

\section{Conclusión}

En definitiva, si es la realidad la que es compleja, es evidente que la complejidad de la que venimos hablando, es la confirmación de dicha realidad. La complejidad no es un invento, sino que emerge del tratamiento que hacemos de la misma realidad, sino que emerge del conocimiento que queremos tener de dicha realidad. Desde esta perspectiva, la complejidad es algo que lo atraviesa todo: la realidad, el conocimiento que podamos adquirir de ella, y la aplicación que podamos hacer del conocimiento que hemos adquirido.

\section{Bibliografía}

J. L. González Quirós, Mente y cerebro. Madrid, Iberediciones, 1994.

K. R. Popper, J. C. Ecles, El yo y su cerebro. Barcelona, Roche, 1980.

K. R. Popper, Conjeturas y refutaciones. Barcelona, Paidós, 1983.

H. Maturana y F. Varela, El árbol del conocimiento. Las bases biológicas del conocimiento humano. Madrid, Debate, 1996.

X. Zubiri, Estructura dinámica de la realidad. Madrid, Alianza, 1989.

M. Bunge, “Azar y casualidad, caos y accidente”. En Cápsulas. Barcelona, Gedisa, 2003.

M. Bunge, La ciencia, su método y su filosofía. Santafé de Bogotá, Panamericana, 1996.

M. Bunge, La investigación científica. Barcelona, Ariel, 1979.

M. Bunge, Emergencia y convergencia. Novedad cualitativa y unidad del conocimiento. Barcelona, Gedisa, 2003.

C. Allégre, La derrota de Platón o la ciencia en el siglo XX. México, FCE, 2003.

I. Prigogine, El nacimiento del tiempo, Barcelona, Tusquets, 1998.

A. R. Luria, El cerebro humano y los procesos psíquicos. Barcelona, Fontanella, 1979. 
J-M. Levy-Leblond, La piedra de toque. La ciencia a prueba. México, FCE, 2004.

F. Martínez Mendoza, Neurociencias y formación inicial. México, Trillas, 2010.

Th. Kuhn. La estructura de las revoluciones científicas. México, FCE, 1971.
A. S. Vázquez, Filosofía de la praxis. México, Grijalbo, 1967.
E. Ferreiro, Cultura escrita y educación. Conversaciones con Emilia Ferreiro. México, FCE, 1999. 